\title{
Antimicrobial profile and organoleptic acceptability of some essentials oils and their blends in hurdle treated chicken meat spread
}

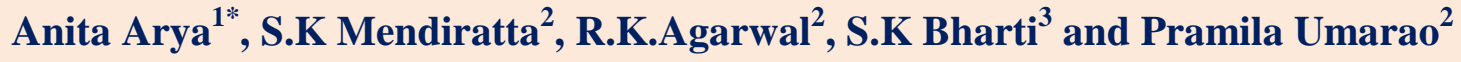 \\ ${ }^{1}$ Department of Livestock Products Technology, College of Veterinary and Animal Sciences \\ GBPUAT, Pantnagar-263145, (Uttarakhand), India \\ ${ }^{2}$ 5ICAR-Indian Veterinary Research Institute, Bareilly, Uttar Pradesh, India \\ ${ }^{3}$ Department of LPT DUVASU, Mathura, Uttar Pradesh, India \\ *Corresponding author
}

\section{A B S T R A C T}

Keywords

Antimicrobial

effect,

Essential oil,

Hurdle treated,

chicken meat spread

Article Info

Accepted:

20 August 2019

Available Online:

10 September 2019
The study was intended to compare the suitability of incorporation of some essential oils and their blends as natural antimicrobials in hurdle treated chicken meat spread with assent to their organoleptic acceptability. In consideration to MIC of Oregano, cassia cinnamon, thyme, clove and holy basil essential oils (EOs) against Staphylococcus aureus and E coli, 0.125, 0.20 and $0.21 \%$ levels were incorporated in chicken meat spread. Chicken meat spread containing basil and clove EOs showed significantly $(\mathrm{P}<0.05)$ lower flavor, aftertaste and overall acceptability scores. Incorporation of holy basil EOs and clove EOs even at $0.125 \%$ level showed significantly reduced sensory acceptability. Holy basil, oregano and clove EOs showed significantly $(\mathrm{P}<0.05)$ higher antimicrobial activity at $0.125 \%, 0.20 \%$ and $0.25 \%$ level respectively, moreover, oregano EO was found to be most effective against yeast and mold count. Out of the 5 EOs blends, only Blend 1 (oregano, cassia, thyme, clove and holy basil and Blend 4 (cassia, clove and holy basil) were sensorically acceptable however, all the blends showed significantly $(\mathrm{P}<0.05)$ higher antimicrobial property.

\section{Introduction}

Essential oils have plausible quality for preservation though their sensory acceptability is a considerable wringer. Spoilage of processed meat product is a financial burden to producers that commence the food technologists to develop advanced methods for extending shelf-life and quality of the meat. The growth of spoilage and food-borne pathogens is one of the most significant causes for food degradation. Synthetic antimicrobial and antioxidant compound may produce negative health impact which can be reduced by natural food additives as reported by Alves-Silva et al. (2013). Extract from spices and herbs have been used for enhancing the organoleptic characteristics as well as shelf life of food products. Essential oils (EOs) are volatile liquids extracted from plant material such as root bark and leave flower, fruit, seed, whole plant or the product of plants secondary 
metabolism Oussalah et al. (2006). Essential oil posses antibacterial, antiparasitic, antifungal, insecticidal and antioxidant properties as described by Viudo-Martos et al. (2010) and Zhang et al. (2016). Major compound of essential oil are phenolic compounds such as monoterpenes (carvacrol, thymol or eugenol, monoterpenic), hydrocarbons (p-cimene, c-terpinene, a-pinene or limonene), alcohol terpenoids (borneol, linalool, 1,8-cineole or geraniol), aldehydes (cinnamaldehyde, geranial or citronnell) and ketones (piperitone or carvone). These components are very volatile and can be easily decomposed in food with effect of high temperature, and pressure. Davidson and Naidu (2000) classified spices and herbs based on antimicrobial activity. Cinnamon, clove, mustard and vanillin are categorized as the spices with strong antimicrobial activity. Basil, oregano, rosemary sage and thyme are the herbs with strong antimicrobial activity. Anise bay, black pepper, cardamom, chilli powder, coriander cumen, curry powder fenugreek, ginger, juniper oil, mace, marjoram, mint, nutmeg, paprika, sesame, spearmint, fenugreek and white pepper spices and herbs with limited antimicrobial activity as classified by Davidson and Naidu (2000). Cinnamon and clove contains cinnamaldehyde and eugenol whereas, major antimicrobial compound of oregano and thyme is carvacrol (62-79\%), and thymol (42\%) respectively. Callaway et al. (2011) observed EOs as effective antimicrobials against different food borne pathogen like E. coli O157:H7, Salmonella typhimurium, $S$. aureus, $L$. monocytogenes, and Campylobacter coli. Mechanism of antimicrobial action attributed due to their lipofilic character and functional group which causes increased bacterial cell membrane permeability as reported by Burt (2004) and Lambert et al. (2001). However essential oils are efficient biopreservatives, considering their negative organoleptic impact, the lowest application concentration should be determined at which they are sensorically acceptable as described by Turgis et al. (2012).

Chicken meat spread is a cooked spreadable, convenience product to be spread on or sandwiched in a base like bread. However, water and fat separation, short shelf life and rancidity are the basic problems associated. Objective of the study is to optimize level of different essential oils and their blends in hurdle treated chicken meat spread as additional hurdle with honey and vinegar for enhancement of microbial quality with consideration to their organoleptic acceptance.

\section{Materials and Methods}

\section{Preparation of Sweet and sour chicken meat spread}

White leghorn layer spent hen of approximately 72-100 weeks was slaughtered using the halal method in the experimental abattoir of division of LPT, IVRI, Izatnagar. Carcasses were manually deboned and conditioned for $24 \mathrm{~h}$ at $4^{\circ} \mathrm{C}$ followed by storage at $-18 \pm 1^{\circ} \mathrm{C}$ till further use. The deboned-frozen meat was thawed overnight in refrigerator and cut into small chunks. The spice ingredients in desired ratio were dried at $50 \pm 2^{\circ} \mathrm{C}$ for $2 \mathrm{~h}$ followed by grinding and sieving through 100 mesh. The formulation contained anise $8 \%$, black pepper $10 \%$, caraway $10 \%$, cardamom $6 \%$, red chili $8 \%$, cloves $3 \%$, cinnamon $6 \%$, cumin $12 \%$, dry ginger $10 \%$, mace $1 \%$, nutmeg $1 \%$ turmeric $10 \%$ and coriander $15 \%(\mathrm{w} / \mathrm{w})$. The spice mix was stored at ambient temperature in a polyethylene terephthalate (PET) container (Godrej Cold Gold, India). For preparation of condiments mix, onion, ginger and garlic were used at $(3: 2: 1)$ ratio and grinded. Tomato powder was prepared in laboratory using prestandardized procedure of Jayathunge et al. (2012) with slight modification. Fresh ripened 
tomatoes were washed and blanched at $60^{\circ} \mathrm{C}$ for $1 \mathrm{~min}$ then sliced into thin pieces of about 5-8 $\mathrm{mm}$. Pieces were subjected to drying in hot air oven at $70^{\circ} \mathrm{C}$ initially followed by drying at $50^{\circ} \mathrm{C}$ for $68 \mathrm{~h}$ with turning in between. Dried tomatoes were pulverized to form powder and packed in laminated pouches.

Meat pieces were grinded in meat grinder (Mado Eskimo Mew 714, Mado, Germany) mixed with the condiments and spices and cooked by braising at $84^{\circ} \mathrm{C}$ for $14 \mathrm{~min}$. At the end of braising, honey (humectants) and vinegar (acidulant) were added followed by addition of chitosan and finally grounded in a chopper to pasty consistency honey $14.36 \%$, vinegar $5.41 \%$ and tomato powder $1.4 \%$ was added on the bases of previous prestandardization trials based on response surface methodology (Arya,2017).

The formulation of sweet and sour chicken meat spread is presented in (table1). Developed product was subjected to product profile analysis for proximate composition, total dietary fibre content, lycopene content $\mathrm{pH}$ and water activity values. The product optimized was incorporated with different essential oils and their blends in the next experiment.

\section{Application of essential oils}

Different essential oils as Oregano, cassia, thyme cinnamon, clove and holy basil were decided to be added in the chicken meat spread containing humectants, acidifier and natural colorant. Meat pieces were divided into different treatment groups and incorporated with different levels of essential oils $(0.125 \%, 0.20 \%$ and $0.25 \%$ ) separately (based on various preliminary trials) by swabbing with sterilized cotton swabs and left covered in desiccators for $30 \mathrm{~min}$ and subjected to further processing as above.

\section{Determination of MIC}

Standard culture of 2 bacterial strains Staphylococcus aureus (AICC15597) and E coli (ATCCBAA977) were taken and one colony of test bacterial strain was transferred into into $5 \mathrm{ml} \mathrm{BHI}$ broth tubes which were incubated at $37^{\circ} \mathrm{C}$ for $24 \mathrm{~h}$. From there tubes $16.66 \mu \mathrm{l}$ was to transferred to another $5 \mathrm{ml}$ BHI broth tubes to make 300 times dilution. $50 \mu \mathrm{l}$ of 300 times diluted broth culture was transferred into tubes containing $5 \mathrm{ml} \mathrm{BHI}$ and Essential oil in increasing order $(0.01$ to $0.1 \%)$ added in order to check MIC and after Incubated at $37^{\circ} \mathrm{C}$ for $24-48 \mathrm{~h}$, all the tubes were checked for turbidity. The experiment was repeated thrice in duplicates and mean values were taken as MIC.

\section{Preparation of EO blends}

Blends of essential oils were prepared by using different essential oil combinations in sterilized vials. Optimized concentration of individual oil was standardized to form blend on the basis of sensory acceptability and antimicrobial effect Different (Table 2) concentrations of different essential oils were optimized in blends in previous trials.

Individual blend containing optimized percentage of essential oil was applied at $0.125 \%$ level in chicken meat spread by swabbing method.

\section{Sensory evaluation}

Sensory attributes for chicken meat spread were evaluated using 8 point descriptive scale Keeton et al. (1983). Where 8 score was given for extremely good and 1 was given for extremely poor. Panellist consisting of scientists and post graduate students of the LPT Division were make familiarized with the nature product without disclosing the identity of the product and also briefed about for the 
product attributes viz color and appearance, flavor, spread ability, texture, after taste, adhesive ability and overall acceptability. Products were evaluated at ambient temperature with and without spreading over a piece of bread. Plain water was provided to rinse the mouth in between the samples.

Proximate composition, total dietary fibre and lycopene content

Proximate composition was analysed as per the method described by AOAC (1995) and Total dietary fiber (TDF) along with soluble and insoluble dietary fiber was determined by slight modification of an enzymatic method given by AOAC (1995). Lycopene content was measured following the method described by Fish et al. (2002) with slight modifications

\section{pH and water activity}

$\mathrm{pH}$ was measured using the digital $\mathrm{pH}$ meter (Cyberscan ${ }^{\circledR}, \mathrm{pH} 510$, Eutech Instruments, Singapore). Water activity was measured with the help of water activity meter (Hygrolab 3®, Rotronics, Switzerland).

\section{Microbiological Evaluation}

Microbiological quality of treatment and control samples were analysed following the methods described by American Public Health Association APHA (1984). Plate count agar, Potato Dextrose Agar and violet red bile agar were respectively used for the Specific plate count, yeast and mold count and coliforms count. Serial dilutions of the samples were made using sterile $0.1 \%$ peptone water and mixed uniformly to get dilutions $10^{-2}, 10^{-3}$ and so on.

After inoculation by pour plate method, plates were kept for $72 \mathrm{hr}$ at $37^{\circ} \mathrm{C}$ for specific plate count, $25^{\circ} \mathrm{C}$ for $5 \mathrm{~d}$ for yeast and mold count and $35 \pm 2{ }^{\circ} \mathrm{C}$ for $48 \mathrm{~h}$ for coliforms counts. Plates showing 30-300 colonies were counted.
The number of colonies was multiplied by the reciprocal of the dilution and expressed as $\log _{10} \mathrm{cfu} / \mathrm{g}$.

\section{Statistical analysis}

Each trial was replicated thrice in duplicate $(n=6)$. The statistical analysis of the data was done through analysis of variance (ANOVA one way analysis technique using SPSS Statistics Software. Differences between means were considered significant when $\mathrm{P}<0.05$. Duncan's multiple range tests were used to detect differences among mean values.

\section{Results and Discussion}

Product profile analysis revealed that the optimized product containing honey, vinegar and tomato powder showed significantly higher $(\mathrm{P}<0.05)$ cooking yield and lower $(\mathrm{P}<0.05) \mathrm{pH}$ values (Table1). Lower $\mathrm{pH}$ values of the product were due to added ingredients as vinegar and honey. Water activity value significantly $(\mathrm{P}<0.05)$ reduced in the optimized product as honey acted as natural humectants. During proximate compositional analysis ash content was significantly higher in the optimized product whereas protein fat and moisture content did not affected significantly $(\mathrm{P}>0.05)$. Total dietary fibre including soluble as well as insoluble dietary fibre were significantly higher $(\mathrm{P}<0.05)$ in the developed product. Lycopene content $(0.11 \pm 0.008(\mathrm{mg} / 100 \mathrm{gm}))$ was only present in the treatment product was contributed by added tomato powder as colorant.

\section{MIC of the essentials oils}

The results of MIC of different essential oils oregano, cassia, thyme, cinnamon, clove and holy basil essential oils against test bacteria Staphylococcus aureus and Escherichia coli are presented in Table 4 and Fig (1). 
Initial screening of EOs for incorporation into optimized product

On the bases of various preliminary trials, it was found that out of 6 essential oil (oregano, cassia, cinnamon, thyme, clove and holy basil) no essential oil was sensorically acceptable above $\quad 0.25 \%$ concentration. Thus concentration below $0.25 \%$ and above the MIC of essential oils were applied as antimicrobial activity is affected by composition, $\mathrm{pH}, \mathrm{a}_{\mathrm{w}}$, and salt level and higher concentration is required in food matrix for antimicrobial effect as described by Angienda and Hill (2011), Hyldgaard et al. (2012) and Radaelli et al. (2016).

Yeast and mold were evaluated till $21^{\text {st }}$ day of storage for comparison as till $7^{\text {th }}$ day no growth were observed so colonies were evaluated at weekly interval.

Effect of EO incorporation on the Sensory properties: Incorporation level $\mathbf{0 . 1 2 5 \%}$

The results of sensory evaluation at $0.125 \%$ EO incorporation level are presented in Table 5. Appearance \& color, spread ability and texture score of control and treated samples did not differ significantly $(\mathrm{P}>0.05)$ Oregano and cassia EOs sowed highest sensory acceptability among all oils tested. However, significantly decreased $(\mathrm{P}<0.05)$ values was observed for flavour, aftertaste and overall acceptability and were lowest for holy basil followed by clove EOs. Flavour score of oregano did not differ significantly $(\mathrm{P}>0.05)$. Aftertaste of holy basil and clove oil showed the lowest score. Overall acceptability was significantly $(\mathrm{P}<0.05)$ higher for oregano and significantly $(\mathrm{P}<0.05)$ lowest $(\mathrm{P}<0.05)$ for holy basil EOs.

\section{Incorporation level $0.20 \%$}

Among different treatments oregano EO showed significantly $(\mathrm{P}<0.05)$ higher values except for spread ability, texture and adhesive ability (Table 6). Lowest flavour score was obtained for chicken spread containing holy basil EO followed by clove EO. Aftertaste score differed significantly $(\mathrm{P}<0.05)$ and highest score was observed for oregano EO. Non significant $(\mathrm{P}>0.05)$ difference was found in aftertaste score of chicken spread containing oregano, and cassia EO. Overall acceptability of all the treatments differed significantly $(\mathrm{P}<0.05)$ and it was highest for oregano and lowest for holy basil EO.

\section{Incorporation level $0.25 \%$}

Results of appearance \& colour, spreadability and texture of $0.25 \%$ level were similar to that of 0.125 and $0.20 \%$ (Table 7). Flavour score of control was significantly $(\mathrm{P}<0.05)$ higher and among treatments and was highest for oregano followed by cassia>thyme> cinnamon $>$ clove $>$ holy basil EOs incorporated products. Significant difference $(\mathrm{P}<0.05)$ was observed in aftertaste score among different EOs of which holy basil and clove EOs obtained lowest score.

Decreased organoleptic acceptability of the essential oil added products might be attributed to pungent flavour volatiles of essential oils. The intense aroma produced by these flavour volatiles, exceed the acceptable threshold level of the product as described by Lv et al. (2018). Organoleptic impact of essential oils should be considered as the use of extract of natural preservatives can alter the taste or exceed acceptable flavour thresholds as suggested by Hsieh et al. (19) and Nazer $e t$ al. (2005). Tsigarida, et al. (2000) did not observed any unacceptable flavour of $0.8 \%$ $(\mathrm{vol} / \mathrm{wt})$ oregano oil treated fillets after storage at $5^{\circ} \mathrm{C}$ and cooking. However Skandamis et al. (2001) reported improved flavour, odour and colour of minced beef treated with $1 \%$ (vol/wt) oregano EO and stored under 
modified atmospheric packaging and vacuum stored at $5^{\circ} \mathrm{C}$.

Significantly reduced microbial growth peeled shrimps during storage at refrigeration temperature without affecting the sensory properties was observed by Arancibia (2014).

Effect of EO incorporation on the microbiological quality of chicken meat spread

\section{Incorporation level $\mathbf{0 . 1 2 5 \%}$}

Significant difference $(\mathrm{P}<0.05)$ was observed for standard plate count and among treatments it was highest for cinnamon and lowest for holy basil oil (Table 5). However, no significant $(\mathrm{P}>0.05)$ difference was observed for SPC of holy basil EO, oregano and clove EOs incorporated products. Results indicated that best antimicrobial effect were obtained with oregano, holy basil and clove treatments with around $0.4 \log$ cfu reduction in microbial count though cinnamon, thyme and cassia reduced to approx. 0.2 and $0.3 \log \mathrm{cfu}$ respectively. Yeast and mold count were not observed till one week in treatments as well as control so analysis were done at weekly interval.

No yeast and mold colonies were observed on $14^{\text {th }}$ day in the product containing oregano and cassia EOs and on $21^{\text {st }}$ day lowest $(\mathrm{P}<0.05)$ count were observed for oregano followed by clove EOs (Table 3).

\section{Incorporation level $\mathbf{0 . 2 0 \%}$}

Significant difference $(\mathrm{P}<0.05)$ was observed for control and treatments (Table 6) for SPC as well as YMC. Lowest SPC was observed for holy basil EOs whereas highest was showed by thyme EOs No significant difference $(\mathrm{P}>0.05)$ was observed among holy basil and Oregano EOs with around $0.45 \mathrm{log}$ reduction in microbial count. Product containing clove EOs showed approximately
$0.4 \log$ reduction in microbial count, whereas cassia, cinnamon and thyme represented 0.3 $\log$ reduction values.

Yeast and mold were not observed till $14^{\text {th }}$ day in oregano cassia and clove EOs containing product and counts were significantly $(\mathrm{P}<0.05)$ lower for oregano EO followed by clove and cassia EO Table 4).

\section{Incorporation level $\mathbf{0 . 2 5 \%}$}

Standard plate count was significantly different $(\mathrm{P}<0.05)$ at $0.25 \%$ incorporation level (Table 7). Among treatments lowest count were obtained for holy basil and oregano EOs followed by clove, cassia, thyme and cinnamon EOs. Oregano, holy basil, clove EOs exhibited no significant difference $(\mathrm{P}>0.05)$. Results showed that oregano, holy basil and clove EOs incorporation in chicken meat spread reduced total plate count values to approximately 0.5- 0.6 Cassia and thyme 0.4 and cinnamon oil put down reduction up to 0.3 $\log$ value.

Yeast and mold were observed on 21 day in all the treatments (Table 5) and concentration dependent microbial inhibition was also observed for the yeast and mold count where oregano EO showed the highest antifungal activity.

Lower SPC in treatments might be attributed to anti-microbial activity of essential oil compounds such as carvacrol, eugenol and thymol as reported by various researchers (Lambert et al. 2001, Jayasena et al. 2013, Calo et al. 2015, Ghabraie et al. 2016). Difference in antimicrobial potential would be related to their respective composition as well functional groups present and interactions between them.

Enhancement of bacteriostatic and fungistatic effect with increased concentration of EOs may be attributed to dose dependent 
mechanism of action of essential oil as reported by Pesavanto et al. (2015). Ibrahium et al. (2013) evaluated efficiency of clove essential oil (CEO) as antioxidant and antimicrobial in cake preservation and enhancement of antimicrobial activity of clove essential oil was observed with increased application concentration observed that .Significantly reduced microbial count of soy edible films incorporated with thyme and oregano EOs during refrigeration storage was also reported by Emiroglu et al. (2010).

Absence of yeast and mold initially till $7^{\text {th }}$ day would be attributed to hurdle effect of honey, vinegar and essential oils. Thomas et al. (2010) reported that hurdles such as low $\mathrm{pH}$, low $\mathrm{a}_{\mathrm{w}}$ and reheating were sufficient to inhibit yeast and mold growth up to day 3, but additional dipping in $1 \% \mathrm{~K}$-sorbate solution inhibited their growth throughout 9 days.
Significantly lower yeast and mold count in chicken breast meat containing pomegranate juice (PJ) and chitosan $(\mathrm{CH})$ coating enriched with Zataria multiflora essential oil (ZEO) during refrigerated storage was also reported by Bazargani-Gilani et al. (2015). A significant reduction of 2 logarithm units in Penicillium italicum was observed by Sánchez-González, et al. (2010) in chitosan films incorporated with bergamot oil content (3:1 $\mathrm{BO}-\mathrm{CH}$ ratio).

Coliforms were not detected at any concentration throughout till 21 day because of cooking of product to an internal temperature of $72^{\circ} \mathrm{C}$, which might have been lethal to the coliforms; good hygienic practices during and after preparation of products and reduced $\mathrm{pH}$ as well water activity of the product.

Table.1 Formulation for Sweet and sour chicken meat spread

\begin{tabular}{|l|c|c|}
\hline $\begin{array}{l}\text { Ingredients } \\
\text { (w/w) }\end{array}$ & Control & Treatment \\
\hline Chicken meat & 57.00 & 57.00 \\
\hline Oil (v/w) & 12.00 & 12.00 \\
\hline Salt & 2.00 & 2.00 \\
\hline Spices & 3.00 & 3.00 \\
\hline Condiments & 5.00 & 5.00 \\
\hline Potato starch & 2.00 & 2.00 \\
\hline STTP & 0.40 & 0.40 \\
\hline Water (v/w) & 19.17 & - \\
\hline Honey & - & 14.36 \\
\hline Vinegar (v/w) & - & 5.40 \\
\hline Tomato & - & 1.40 \\
\hline powder & 102.57 & 102.57 \\
\hline Total & & \\
\hline
\end{tabular}


Table.2 Composition of essential oil blend

\begin{tabular}{|c|c|c|c|c|c|}
\hline Essential oils (\%) & Blend-1 & Blend-2 & Blend -3 & Blend- 4 & Blend-5 \\
\hline Oregano & 40 & 75 & - & - & - \\
\hline Cassia & 30 & 25 & 40 & 40 & 20 \\
\hline Thyme & 10 & - & 20 & 40 & 15 \\
\hline Clove & 10 & - & 20 & 20 & 20 \\
\hline Holy basil & 10 & - & 20 & - & 25 \\
\hline Ajovan & - & - & - & - & 10 \\
\hline Beetal & - & - & - & - & 10 \\
\hline
\end{tabular}

Table.3 Product profile

\begin{tabular}{|l|l|l|}
\hline Attributes & $\begin{array}{l}\text { Control (without } \\
\text { honey, vinegar and } \\
\text { tomato powder) }\end{array}$ & Treatment product \\
\hline Cooking yield & $83.5 \pm 0.428^{\mathrm{b}}$ & $86.66 \pm 0.421^{\mathrm{a}}$ \\
\hline pH & $6.38 . \pm 094^{\mathrm{a}}$ & $5.00 \pm 0.085^{\mathrm{b}}$ \\
\hline $\mathbf{a}_{\mathbf{w}}$ & $0.96 \pm .006^{\mathrm{a}}$ & $0.88 \pm .004^{\mathrm{b}}$ \\
\hline moisture & $52.83 \pm 0.654^{\mathrm{a}}$ & $46.66 \pm 0.494^{\mathrm{a}}$ \\
\hline pr & $11.73 \pm 0.445^{\mathrm{a}}$ & $10.74 \pm 0.386^{\mathrm{a}}$ \\
\hline fat & $13.05 \pm 0.192^{\mathrm{a}}$ & $13.05 \pm 0.43^{\mathrm{a}}$ \\
\hline ash & $2.41 \pm 0.011^{\mathrm{b}}$ & $2.51 \pm 0.023^{\mathrm{a}}$ \\
\hline IDF & $0.20 \pm 0.004^{\mathrm{b}}$ & $0.40 \pm 0.007^{\mathrm{a}}$ \\
\hline SDF & $0.07 \pm 0.004^{\mathrm{b}}$ & $0.12 \pm 0.006^{\mathrm{a}}$ \\
\hline TDF & $0.28 \pm 0.005^{\mathrm{b}}$ & $0.53 \pm 0.013^{\mathrm{a}}$ \\
\hline Lycopene \\
(mg/100gm) & $0 \pm 0$ & $0.11 \pm 0.008^{\mathrm{a}}$ \\
\hline
\end{tabular}

$\mathrm{n}=6, \quad$ Mean \pm S.E. bearing different superscripts row wise (differ significantly $(\mathrm{P}<0.05)$

Table.4 MIC of essentials oils against test bacteria

\begin{tabular}{|l|c|c|}
\hline Essentials oils & Escherichia coli & $\begin{array}{c}\text { Staphylococcus } \\
\text { aureus }\end{array}$ \\
\hline Oregano & 0.05 & 0.03 \\
\hline Cassia & 0.04 & 0.10 \\
\hline Thyme & 0.13 & 0.25 \\
\hline Cinnamon & 0.23 & 0.25 \\
\hline Clove & 0.04 & 0.06 \\
\hline Holy basil & 0.06 & 0.04 \\
\hline
\end{tabular}


Table.5 Sensory attributes and SPC $\left(\log _{10} \mathrm{cfu} / \mathrm{g}\right), \mathrm{YMC}$, water activity and $\mathrm{pH}$ values of essential oil incorporated $(0.125 \%)$ chicken meat spread

\begin{tabular}{|c|c|c|c|c|c|c|c|c|}
\hline \multirow{2}{*}{\multicolumn{2}{|c|}{$\begin{array}{l}\text { Appearance } \\
\text { and color }\end{array}$}} & Con & Oregano & Cassia & Cinnamon & Thyme & Clove & Holy basil \\
\hline & & $7.31 \pm 04^{\mathrm{a}}$ & $7.4 \pm 0.04^{\mathrm{a}}$ & $7.28 \pm .05^{\mathrm{a}}$ & $7.42 \pm 0.05^{\mathrm{a}}$ & $7.35 \pm 0.02^{\mathrm{a}}$ & $7.35 \pm 0.04^{\mathrm{a}}$ & $7.43 \pm 0.04^{\mathrm{a}}$ \\
\hline \multicolumn{2}{|c|}{ Flavor } & $7.31 \pm 0.03^{\mathrm{a}}$ & $7.21 \pm 0.03^{b}$ & $7.05 \pm 0.04^{c}$ & $6.55 \pm 0.02^{\mathrm{e}}$ & $6.83 \pm 0.07^{\mathrm{dc}}$ & $6.35 \pm 0.17^{\mathrm{e}}$ & $4.98 \pm 0.14^{\mathrm{f}}$ \\
\hline \multicolumn{2}{|c|}{ Spradability } & $7.31 \pm 0.02^{\mathrm{a}}$ & $7.28 \pm 0.05^{\mathrm{a}}$ & $7.18 \pm 0.05^{\mathrm{a}}$ & $7.18 \pm 0.04^{\mathrm{a}}$ & $7.18 \pm 0.05^{\mathrm{a}}$ & $7.21 \pm 0.05^{\mathrm{a}}$ & $7.28 \pm 0.06^{\mathrm{a}}$ \\
\hline \multicolumn{2}{|c|}{ Texture } & $7.35 \pm 0.04^{\mathrm{a}}$. & $7.3 \pm 0.06^{\mathrm{a}}$ & $7.35 \pm 0.04^{\mathrm{a}}$ & $7.3 \pm 0.06^{\mathrm{a}}$ & $7.36 \pm 0.04^{\mathrm{a}}$ & $7.3 \pm 0.06^{\mathrm{a}}$ & $7.36 \pm 0.04^{\mathrm{a}}$ \\
\hline \multicolumn{2}{|c|}{ Aftertaste } & $7.38 \pm .04^{\mathrm{a}}$ & $7.31 \pm 0.05^{\mathrm{a}}$ & $7.15 \pm 0.03^{b}$ & $6.88 \pm 0.05^{c}$ & $6.8 \pm 0.06^{c}$ & $6.58 \pm 0.05^{\mathrm{d}}$ & $5.56 \pm 0.07^{\mathrm{e}}$ \\
\hline \multicolumn{2}{|c|}{$\begin{array}{l}\text { Adhesive } \\
\text { ability }\end{array}$} & $7.36 \pm 0.03^{\mathrm{a}}$ & $7.38 \pm 0.03^{\mathrm{a}}$ & $7.28 \pm 0.06^{\mathrm{a}}$ & $7.33 \pm 0.04^{\mathrm{a}}$ & $7.35 \pm 0.04^{\mathrm{a}}$ & $6.78 \pm 0.06^{\mathrm{a}}$ & $7.31 \pm 0.03^{\mathrm{a}}$ \\
\hline \multicolumn{2}{|c|}{$\begin{array}{l}\text { Overall } \\
\text { acceptibility }\end{array}$} & $7.33 \pm 0.03^{\mathrm{a}}$ & $7.21 \pm 0.03^{b c}$ & $7.06 \pm 0.03^{c}$ & $6.7 \pm 0.079^{d}$ & $6.8 \pm 0.036^{\mathrm{d}}$ & $6.4 \pm 0.12^{\mathrm{e}}$ & $5.35 \pm 0.056^{\mathrm{f}}$ \\
\hline \multicolumn{2}{|c|}{ SPC } & $2.22 \pm 0.02^{\mathrm{a}}$ & $1.83 \pm 0.02^{\mathrm{cb}}$ & $1.93 \pm 0.01^{\mathrm{b}}$ & $1.98 \pm 0.03^{\mathrm{b}}$ & $1.99 \pm 0.03^{\mathrm{b}}$ & $1.89 \pm 0.028^{\mathrm{cb}}$ & $1.82 \pm 0.03^{\mathrm{cb}}$ \\
\hline \multirow[t]{4}{*}{$\mathbf{Y} \& \mathbf{M}$} & 0 & ND & ND & ND & ND & ND & ND & ND \\
\hline & 7 & ND & ND & ND & ND & ND & ND & ND \\
\hline & 14 & $1.22 \pm 0.16^{\mathrm{a}}$ & ND & ND & $0.92 \pm 0.04^{d}$ & $1.01 \pm 0.03^{c}$ & $0.63 \pm 0.04^{\mathrm{e}}$ & $1.09 \pm 0.06^{b}$ \\
\hline & 21 & $1.41 \pm 0.03^{\mathrm{a}}$ & $0.92 \pm 0.01^{\mathrm{e}}$ & $1.06 \pm 0.04^{\mathrm{c}}$ & $1.02 \pm 0.02^{\mathrm{cd}}$ & $1.21 \pm 0.03^{b}$ & $0.96 \pm 0.03^{\mathrm{d}}$ & $1.09 \pm 0.06^{b}$ \\
\hline \multicolumn{2}{|l|}{$\mathbf{a}_{\mathbf{w}}$} & $0.90 \pm 0.004$ & $0.89 \pm 0.006$ & $0.89 \pm 0.006$ & $0.90 \pm 0.01$ & $0.90 \pm .004$ & $0.88 \pm 0.01$ & $0.90 \pm 0.01$ \\
\hline \multicolumn{2}{|l|}{ pH } & $4.88 \pm 0.05$ & $4.34 \pm 0.05$ & $4.85 \pm 0.06$ & $4.83 \pm 0.017$ & $5.05 \pm 0.16$ & $4.75 \pm 0.06$ & $4.80 \pm 0.08$ \\
\hline
\end{tabular}

$\mathrm{n}=21, \mathrm{n}=6(\mathrm{TPC})$. Mean \pm S.E. bearing different superscripts row wise (differ significantly $(\mathrm{P}<0.05)$

Table.6 Sensory attributes and SPC ( $\left.\log _{10} \mathrm{cfu} / \mathrm{g}\right)$, YMC, water activity and $\mathrm{pH}$ values of of essential oil incorporated $(0.20 \%)$ chicken meat spread.

\begin{tabular}{|c|c|c|c|c|c|c|c|c|}
\hline & Con & Oregano & Cassia & Cinnamon & Thyme & Clove & Holy basil \\
\hline \multicolumn{2}{|c|}{ Appearance } & $7.38 \pm 0.03$ & $7.5 \pm 0$ & $7.36 \pm 0.08$ & $7.35 \pm 0.04$ & $7.18 \pm 0.05$ & $7.3 \pm 0.04$ & $7.25 \pm 0.07$ \\
\hline \multicolumn{2}{|c|}{ Flavor } & $7.31 \pm 0.03^{\mathrm{a}}$ & $7 \pm 0.025^{b c}$ & $6.88 \pm 0.03^{c}$ & $6.67 \pm 0.04^{\mathrm{de}}$ & $6.93 \pm 0.07^{\mathrm{e}}$ & $5.25 \pm 0.105^{\mathrm{f}}$ & $4.88 \pm 0.087^{\mathrm{g}}$ \\
\hline \multicolumn{2}{|c|}{ Spreadability } & $7.35 \pm 0.04$ & $7.26 \pm 0.06$ & $7.33 \pm 0.03$ & $7.33 \pm 0.05$ & $7.36 \pm 0.02$ & $7.25 \pm 0.03$ & $7.35 \pm 0.02$ \\
\hline \multicolumn{2}{|c|}{ Texture } & $6.25 \pm 1.15$ & $16.93 \pm 11.07$ & $7.3 \pm 0.09$ & $7.41 \pm 0.06$ & $7.05 \pm 0.22$ & $7.36 \pm 0.05$ & $7.33 \pm 0.08$ \\
\hline \multicolumn{2}{|c|}{ Aftertaste } & $7.38 \pm 0.03^{\mathrm{a}}$ & $6.95 \pm 0.04^{\mathrm{bc}}$ & $6.58 \pm 0.16^{\mathrm{c}}$ & $5.81 \pm 0.09^{\mathrm{de}}$ & $5.8 \pm 0.07^{\mathrm{e}}$ & $5.15 \pm 0.10^{\mathrm{fg}}$ & $4.8 \pm 0.08^{\mathrm{g}}$ \\
\hline \multicolumn{2}{|c|}{ Adhesiveabilit } & $7.31 \pm 0.07$ & $7.43 \pm 0.05$ & $7.43 \pm 0.04$ & $7.36 \pm 0.05$ & $7.4 \pm 0.05$ & $7.32 \pm 0.05$ & $7.45 \pm 0.04$ \\
\hline \multicolumn{2}{|c|}{$\begin{array}{l}\text { Overall } \\
\text { acceptibility }\end{array}$} & $7.21 \pm 0.03^{\mathrm{a}}$ & $7.13 \pm 0.03^{\mathrm{a}}$ & $7.08 \pm 0.04^{\mathrm{a}}$ & $6.23 \pm 0.08^{c}$ & $6.81 \pm 0.04^{\mathrm{b}}$ & $5.73 \pm 0.05^{\mathrm{d}}$ & $4.98 \pm 0.047^{\mathrm{e}}$ \\
\hline \multicolumn{2}{|c|}{ TPC } & $2.24 \pm 0.01^{\mathrm{a}}$ & $1.76 \pm 0.01^{\mathrm{d}}$ & $1.87 \pm 0.03^{\mathrm{c}}$ & $1.97 \pm 0.03^{\mathrm{b}}$ & $1.91 \pm 0.03^{\mathrm{c}}$ & $1.81 \pm 0.03^{\mathrm{d}}$ & $1.75 \pm 0.03^{\mathrm{e}}$ \\
\hline \multirow[t]{4}{*}{ YMC } & 0 & ND & ND & ND & ND & ND & ND & ND \\
\hline & 7 & ND & ND & ND & ND & ND & ND & ND \\
\hline & 14 & $1.24 \pm 0.02^{\mathrm{a}}$ & ND & ND & $0.53 \pm 0.20^{\mathrm{d}}$ & $0.60 \pm 0.02^{c}$ & ND & $0.92 \pm 0.02^{b}$ \\
\hline & 21 & $1.39 \pm 0.01^{\mathrm{a}}$ & $0.73 \pm 0.01^{\mathrm{ed}}$ & $0.84 \pm 0.02^{c}$ & $0.84 \pm 0.02^{\mathrm{c}}$ & $0.96 \pm 0.004^{b}$ & $0.75 \pm 0.03^{d}$ & $1.18 \pm 0.03$ \\
\hline & $0.89 \pm 0.006$ & $0.89 \pm 0.005$ & $0.87 \pm 0.005$ & $0.90 \pm 0.005$ & $0.88 \pm 0.006$ & $0.89 \pm 0.004$ & $0.88 \pm 0.01$ \\
\hline & pH & $4.95 \pm 0.08$ & $4.88 \pm 0.06$ & $4.89 \pm 0.10$ & $4.86 \pm 0.08$ & $4.90 \pm 0.05$ & $4.56 \pm 0.20$ & $4.98 \pm 0.10$ \\
\hline
\end{tabular}

$\mathrm{n}=21, \mathrm{n}=6(\mathrm{TPC})$. Mean \pm S.E. bearing different superscripts row wise (differ significantly $(\mathrm{P}<0.05)$ 
Table.7 Sensory attributes and SPC $\left(\log _{10} \mathrm{cfu} / \mathrm{g}\right)$, YMC, water activity and $\mathrm{pH}$ values of of essential oil incorporated $(0.25 \%)$ chicken meat spread

\begin{tabular}{|c|c|c|c|c|c|c|c|c|}
\hline & Con & Oregano & Cassia & Cinnamon & Thyme & Clove & Holy basil \\
\hline \multicolumn{2}{|c|}{ Appearance } & $7.46 \pm 0.03$ & $7.31 \pm 0.07$ & $7.36 \pm 0.03$ & $7.4 \pm 0.03$ & $7.41 \pm 0.02$ & $7.38 \pm 0.04$ & $7.45 \pm 0.02^{\mathrm{a}}$ \\
\hline \multicolumn{2}{|c|}{ Flavor } & $7.45 \pm 0.04$ & $6.71 \pm 0.07$ & $6.53 \pm 0.03$ & $6.35 \pm 0.06$ & $6.58 \pm 0.04$ & $5.4 \pm 0.03$ & $4.58 \pm 0.08$ \\
\hline \multicolumn{2}{|c|}{ Spradability } & $7.28 \pm 0.03^{\mathrm{a}}$ & $7.48 \pm 0.03^{\mathrm{a}}$ & $7.46 \pm 0.04^{\mathrm{a}}$ & $7.38 \pm 0.08^{\mathrm{a}}$ & $7.51 \pm 0.04^{\mathrm{a}}$ & $7.36 \pm 0.03^{\mathrm{a}}$ & $7.48 \pm 0.03^{\mathrm{a}}$ \\
\hline \multicolumn{2}{|c|}{ Texture } & $7.35 \pm 0.02^{\mathrm{a}}$ & $7.32 \pm 0.03^{\mathrm{a}}$ & $7.35 \pm 0.02^{\mathrm{a}}$ & $7.35 \pm 0.04^{\mathrm{a}}$ & $7.43 \pm 0.04^{\mathrm{a}}$ & $7.45 \pm 0.03^{\mathrm{a}}$ & $7.41 \pm 0.04^{\mathrm{a}}$ \\
\hline \multicolumn{2}{|c|}{ Aftertaste } & $7.28 \pm 0.06^{\mathrm{a}}$ & $6.88 \pm 0.04^{\mathrm{bc}}$ & $6.86 \pm 0.04^{c}$ & $6.06 \pm 0.06^{\mathrm{d}}$ & $6.28 \pm 0.07^{\mathrm{d}}$ & $4.83 \pm 0.12^{\mathrm{e}}$ & $4.68 \pm 0.06^{t}$ \\
\hline \multicolumn{2}{|c|}{ Adhesiveabilit } & $0.04^{\mathrm{a}}$ & $7.48 \pm 0.03^{\mathrm{a}}$ & $7.45 \pm 0.03^{\mathrm{a}}$ & $7.38 \pm 0.03^{\mathrm{a}}$ & $7.45 \pm 0.04^{\mathrm{a}}$ & $7.41 \pm 0.03^{\mathrm{a}}$ & $7.38 \pm 0.04^{\mathrm{a}}$ \\
\hline \multicolumn{2}{|c|}{$\begin{array}{l}\text { Overall } \\
\text { acceptibility }\end{array}$} & $7.26 \pm 0.02^{\mathrm{a}}$ & $6.78 \pm 0.07^{b}$ & $6.51 \pm 0.03^{c}$ & $6.21 \pm 0.07^{d}$ & $6.53 \pm 0.049^{c}$ & $5.25 \pm 0.034^{\mathrm{e}}$ & $4.58 \pm 0.04^{\mathrm{t}}$ \\
\hline \multicolumn{2}{|c|}{ TPC } & $2.23 \pm 0.02^{\mathrm{a}}$ & $1.6 \pm 0.03^{\mathrm{f}}$ & $1.77 \pm 0.02^{\mathrm{d}}$ & $1.94 \pm 0.02^{b}$ & $1.82 \pm 0.0164^{\text {cd }}$ & $1.69 \pm 0.04^{\mathrm{ef}}$ & $1.62 \pm 0.03^{\mathrm{f}}$ \\
\hline \multirow[t]{4}{*}{ YMC } & 0 & ND & ND & ND & ND & ND & ND & ND \\
\hline & 7 & & ND & ND & ND & ND & ND & ND \\
\hline & 14 & $1.23 \pm 0.13$ & ND & ND & ND & ND & ND & ND \\
\hline & 21 & $1.34 \pm 0.02^{\mathrm{a}}$ & $0.54 \pm 0.03^{\mathrm{f}}$ & $0.67 \pm 0.01^{\mathrm{ed}}$ & $0.75 \pm 0.04^{d}$ & $0.83 \pm 0.02^{\mathrm{c}}$ & $0.71 \pm 0.04^{\mathrm{d}}$ & $1.13 \pm 0.03^{b}$ \\
\hline \multicolumn{2}{|l|}{$\mathbf{a}_{\mathbf{w}}$} & $0.90 \pm 0.004$ & $0.87 \pm 0.01$ & $0.90 \pm 0.004$ & $0.88 \pm 0.006$ & $0.89 \pm 0.007$ & $0.90 \pm 0.007$ & $0.90 \pm 0.003$ \\
\hline \multicolumn{2}{|l|}{ pH } & $5.00 \pm 0.06$ & $4.94 \pm 0.11$ & $4.97 \pm 0.09$ & $5.00 \pm 0.06$ & $4.95 \pm 0.06$ & $4.89 \pm 0.06$ & $4.96 \pm 0.02$ \\
\hline
\end{tabular}

$\mathrm{n}=21, \mathrm{n}=6(\mathrm{TPC})$. Mean \pm S.E. bearing different superscripts row wise (differ significantly $(\mathrm{P}<0.05)$

Table.8 Sensory attributes and SPC $\left(\log _{10} \mathrm{cfu} / \mathrm{g}\right), \mathrm{YMC}$, water activity and $\mathrm{pH}$ values of of essential oil blends incorporated $(0.125 \%)$ chicken meat spread

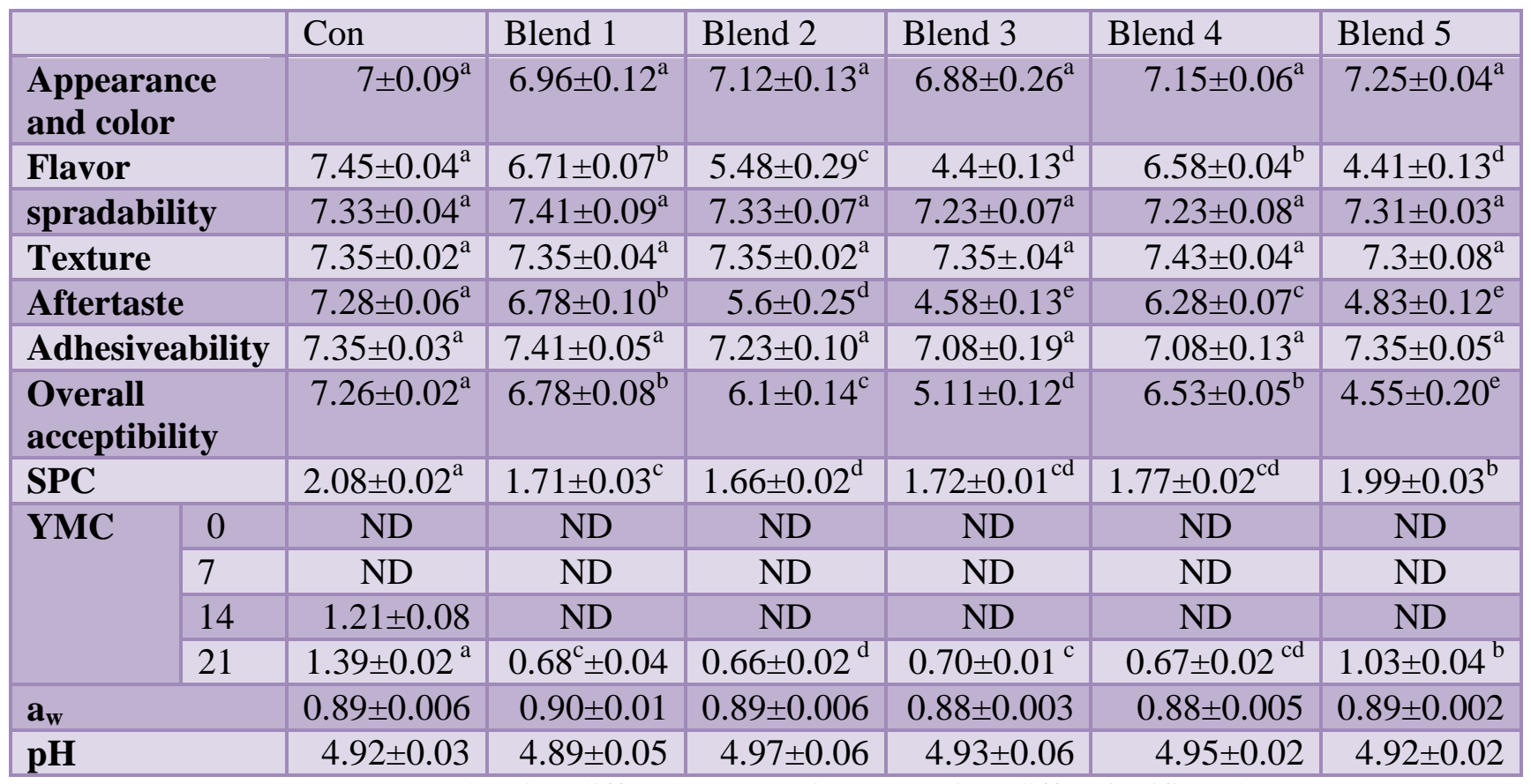

$\mathrm{n}=21, \mathrm{n}=6(\mathrm{TPC})$. Mean \pm S.E. bearing different superscripts row wise (differ significantly $(\mathrm{P}<0.05)$ 


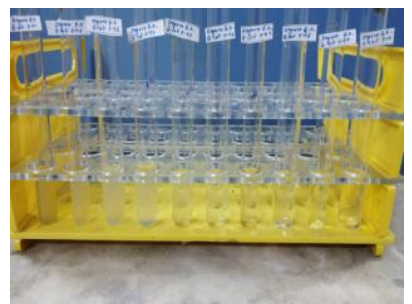

a

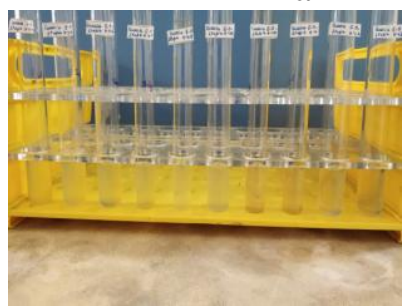

d

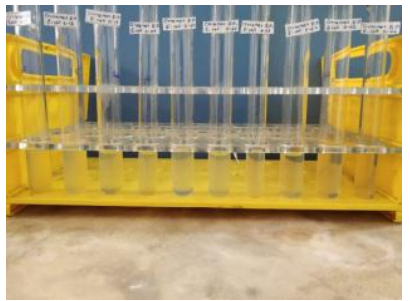

g

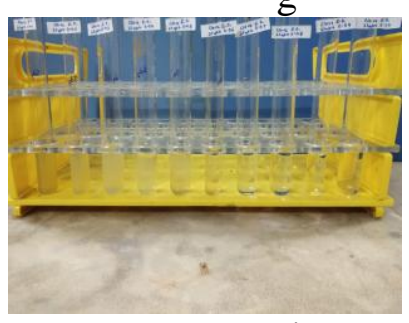

j

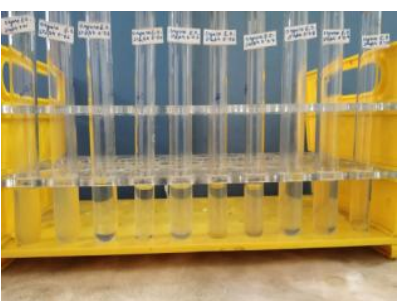

b

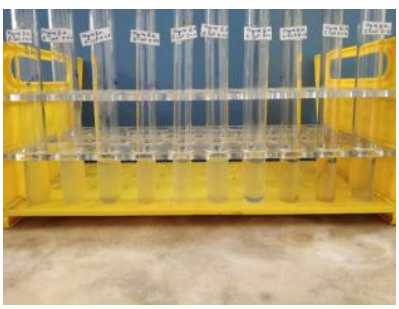

e

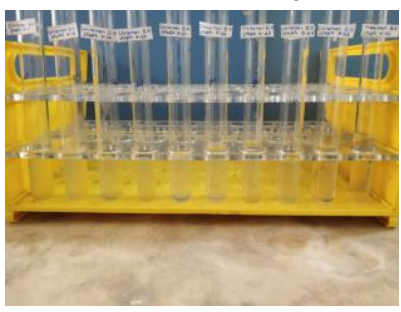

h

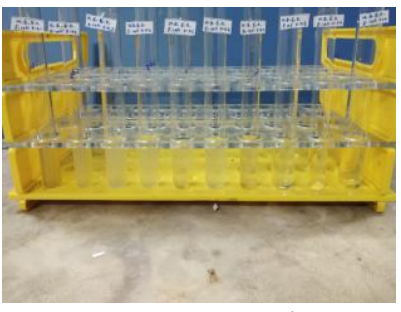

$\mathrm{k}$

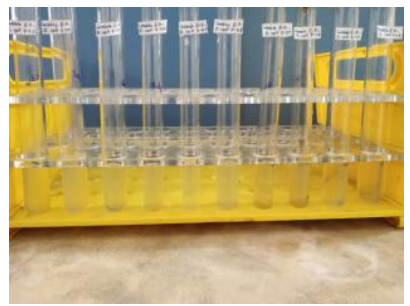

$\mathrm{C}$

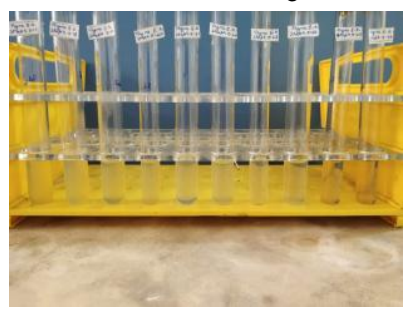

f

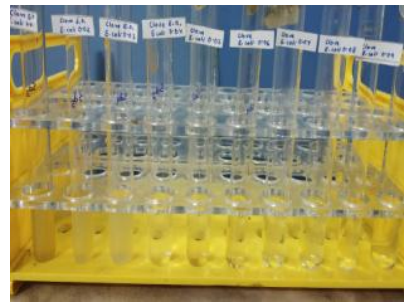

$\mathrm{i}$

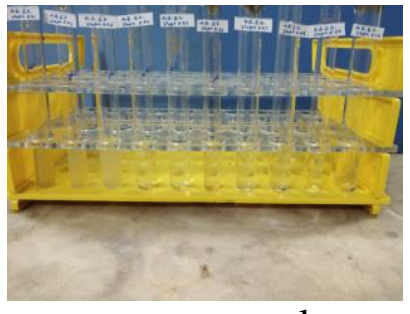

1

Fig.1 Tube dilution test showing the MC of Oregano (a,b),Cassia (c,d),Cinnamon (e,f) Thyme (g,h) Clove (I, j) Holy basil(k,l) against test bacterial culture of Staphylococcus aureus and Escherichia coli

Effect of EO incorporation on the $\mathrm{pH}$ and water activity values of chicken meat spread

$\mathrm{pH}$ and water activity values did not affected by incorporation of essential oils and their blends.

Effect of EO blends incorporation on the Sensory properties of chicken meat spread

The results of sensory evaluation data revealed that non-significant $(\mathrm{P}>0.05)$ difference was observed between treatments and control for spread ability, texture and adhesive ability (Table 8). Flavour score showed significant $(\mathrm{P}<0.05)$ difference between treatment and control. Among the treatments, score of blend 1 was highest followed by blend 4, blend 2 and blend 5. Flavour of blend 3 was significantly $(\mathrm{P}<0.05)$ lower than bland 1 and 4 whereas no significant difference was found between flavour of blend 3 and 5 . After taste score of control and treatment differed significantly whereas among the treatments it was highest for blend 1 and lowest for blend 3 . Overall acceptability of blend 1 was highest followed by blend 4 . 
However studies regarding EO combinations to obtain effective antimicrobial activity at sufficiently lower concentrations with satisfactory sensory quality are scantly available (Delaquis et al. (2002), lv et al. (2002) Gutierrez et al. (2008) and Ghabraie et al. (2016)

Gutierrez et al. (2008) reported that (EOs) in combination can minimize application concentrations and consequently reduce any adverse sensory impact in food. Ghabraie, ((2016) found acceptable smell and taste of ready to cook (RTC) ground beef, added with $0.05 \% \quad(\mathrm{v} / \mathrm{v})$ combined of EOs (Chinese cinnamon and cinnamon bark). However, in present study organoleptic quality significantly decreased by incorporation of blends. Pasavnto et al. (2015) assessed the inhibitory effects of the essential oils (EOs) from oregano and roremarry, as well as its individual constituents (ICs) carvacrol (CAR) and 1,8-cineole (CIN), respectively (combined at subinhibitory concentrations) against a cocktail of Staphylococcus aureus. The incorporation at different combinations in caused a decrease $(P \leq 0.05)$ of initial counts of $S$. aureus in cheese and meat broths. Van Haute et al. (2016) studied the effect of cinnamon, oregano and thyme essential oils in marinade on the microbial shelf life of fish and meat products and showed that the addition of essential oil in marinade considerably affect the sensorial properties.

\section{Effect of EO blends incorporation on the microbial count of chicken meat spread}

The results of microbiological evaluation for total plate count are presented in Table 8 . Total plate count of control was significantly $(\mathrm{P}<0.05)$ higher than all the treatments. Among the treatments count was highest for blend 6 and no significant $(\mathrm{P}>0.05)$ different was observed for count of blend 2, 3 and 4 . Incorporation of blend 2, 3 and 4 at $0.125 \%$ concentration brought about 0.4 to 0.5 and blend 5 brought about $0.2 \log$ reductions in standard plate count value. Yeast and mold count were not observed till one week in treatments as well as control and lowest $(\mathrm{P}<0.05)$ count were observed for blend 2 followed by blend 4 and 3 .

Antibacterial as well as antifungal of effect of blends may be because of synergism/interaction of antimicrobial component of essential oil. Antimicrobial spectrum of an antimicrobial compound can be enhanced if combined together to a suitable concentration as described by Van-Haute $e t a l$. (2016). Some bacteria such as pseudomonas species (showed high resistance to plant antimicrobials can be controlled with combination of antimicrobial as reported by Hammer et al. (1999) and Holley and Patel (2005).

Fei et al. (2011) analysed combined Antibacterial effect of cinnamon, thyme and clove oil against some selected bacteria. Combination of cinnamon and thyme oil showed additive effect against all selected bacteria, and that of cinnamon and clove oil displayed an additive effect against $B$. subtilis, $B$. cereus, $S$. aureus, and an indifferent effect against $E$. coli and $S$. Typhimurium. Thanissery and Smith (2014) reported that combination of EOs exhibited addition effect of antimicrobials for reducing the microorganism by incorporating combination of $0.05 \%$ level of thyme and orange oil in a marinade for inhibiting the Enteritidis and Campylobacter coli numbers on broiler breast fillets and whole wings marinated by vacuum tumbling. Ghabraie et al., 2016) assessed the antibacterial activity of combination of Essential Oils (EOs) against four pathogenic bacteria i.e. Escherichia coli, Listeria monocytogenes, Staphylococcus aureus, and Salmonella typhimurium as well as spoilage bacteria at i.e. Pseudomonas aeruginosa). Chinese cinnamon and Cinnamon bark EOs showed additive antibacterial effects against all bacteria. 
Lv et al. (2011) examined the effectiveness of plant essential oil combinations against four food-related microorganisms. The results stated that four kinds of essential oil combinations showed synergistic antimicrobial activity i.e. oregano-basil for $E$. coli, basil-bergamot for Staphylococcus aureus, oregano-bergamot for B. subtilis and oregano-perilla for $S$. cerevisiae. Antifungal activity of combination of clove and rosemary essential oil was also observed by $\mathrm{Fu}$ et al. (2007)) against Candida albicans.

As per the findings Gutierrez, (2008) oregano combination with thyme can be potential alternative for control of pathogens as well as microbial spoilage, whereas the combinations of oregano marjoram for Gram-negative and thyme sage for control of Gram-positive.

Coliforms were not observed in treatments as well as control till 21 day.

Effect of EO incorporation on the $\mathrm{pH}$ and water activity values of chicken meat spread

$\mathrm{pH}$ and water activity values did not affected by incorporation of essential oil blends.

Study concluded that $0.25 \%$ of oregano and cassia and $0.125 \%$ holy basil and clove EOs were found to be optimum for enhancement of microbial quality of chicken meat spread without much affecting the sensory quality. Antimicrobial effect of oregano, holy basil and clove EOs were high in the product and were almost comparable. At concentration more than $0.125 \%$ holy basil and clove EOs considerably decreased organoleptic quality. Out of the 5 blend used in the study blend, containing oregano, cassia, thyme clove and holy basil and another blend containing cassia, clove and holy basil showed synergistic effect of antimicrobial components. However blend formulation significantly decreased the organoleptic quality rather than neglecting their negative flavour impact. So Essential oils could a good alternative for eradicating spoilage bacteria in chicken meat spread at lower concentration with minimally affecting sensory characteristics meat spread. It be concluded that essential oils as well as their blends can be good choice for replacing synthetic antimicrobials for enhancing of microbial quality of chicken meat spread.

\section{Acknowledgments}

The authors are obliged for providing necessary facilities and funds to carry out the investigation by the Director, Joint Director (Research) and Joint Director (Academics) of Indian Veterinary Research Institute. Research was funded by project Grant no. IVRI/LPT/13-16/005.

\section{References}

Alves-Silva, J. M., dos Santos, S. M. D., Pintado, M. E., Pérez-Álvarez, J. A., Fernández-López, J., \& Viuda-Martos, M. (2013). Chemical composition and in vitro antimicrobial, antifungal and antioxidant properties of essential oils obtained from some herbs widely used in Portugal. Food Control, 32(2), 371-378. E. Sánchez, S. García and N. Heredia, Extracts of edible and medicinal plants damage membranes of Vibrio cholerae. Applied Environmental Microbiology, 76(20), 6888-6894 (2010).

Angienda, P. O., \& Hill, D. J. (2011). The effect of sodium chloride and $\mathrm{pH}$ on the antimicrobial effectiveness of essential oils against pathogenic and food spoilage bacteria: implications in food safety. WASET, 57, 1033-1038.

Arya, A., Mendiratta, S. K., Singh, T. P., Agarwal, R., \& Bharti, S. K. (2017). Development of sweet and sour chicken meat spread based on sensory attributes: process optimization using response 
surface methodology. Journal of food science and technology, 54(13), 42204228.

AOAC 1995. Official Method of Analysis. 16 edn. Association of Official Analytical Chemists, Washington, DC.

APHA. (1984). Compendium of Methods for the Microbiological Examination of Foods $2^{\text {nd }}$ ed. (ed. M.L. Speck). American Public Health Association, Washington, DC

Arancibia, M., Giménez, B., López-Caballero, M. E., Gómez-Guillén, M. C., \& Montero, P. (2014). Release of cinnamon essential oil from polysaccharide bilayer films and its use for microbial growth inhibition in chilled shrimps. LWT-Food Science and Technology, 59(2), 989-995.

Bazargani-Gilani, B., Aliakbarlu, J., \& Tajik, H. (2015). Effect of pomegranate juice dipping and chitosan coating enriched with Zataria multiflora Boiss essential oil on the shelf-life of chicken meat during refrigerated storage. Innovative Food Science \& Emerging Technologies, 29, 280-287.

Burt, S. (2004). Essential oils: their antibacterial properties and potential applications in foods - a review. International journal of food microbiology, 94(3), 223-253.

Callaway, T. R., Carroll, J. A., Arthington, J. D., Edrington, T. S., Anderson, R. C., Ricke, S. C., ... \& Nisbet, D. J. (2011). Citrus products and their use against bacteria: potential health and cost benefits. In Nutrients, Dietary Supplements, and Nutriceuticals (pp. 277-286). Humana Press.

Calo, J. R., Crandall, P. G., O'Bryan, C. A., \& Ricke, S. C. (2015). Essential oils as antimicrobials in food systems-A review. Food Control, 54, 111-119.

Davidson, P. M., \& Naidu, A. S. (2000). Phyto-phenols. InNatural food antimicrobial systems (pp. 278-307). CRC Press.

Delaquis, P. J., Stanich, K., Girard, B., \& Mazza, G. (2002). Antimicrobial activity of individual and mixed fractions of dill, cilantro, coriander and eucalyptus essential oils. International journal of food microbiology, 74(1-2), 101-109.

Emiroğlu, Z. K., Yemiş, G. P., Coşkun, B. K., \& Candoğan, K. (2010). Antimicrobial activity of soy edible films incorporated with thyme and oregano essential oils on fresh ground beef patties. Meat science, 86(2), 283-288.

Fei, L. U., Ding, Y. C., Ye, X. Q., \& Ding, Y. T. (2011). Antibacterial effect of cinnamon oil combined with thyme or clove oil. Agricultural Sciences in China, 10(9), 1482-1487.

Fish, W. W., Perkins-Veazie, P., \& Collins, J. K. (2002). A quantitative assay for lycopene that utilizes reduced volumes of organic solvents. Journal of food composition and analysis, 15(3), 309317.

Fu, Y., Zu, Y., Chen, L., Shi, X., Wang, Z., Sun, S., \& Efferth, T. (2007). Antimicrobial activity of clove and rosemary essential oils alone and in combination. Phytotherapy

Research, 21(10), 989-994.

Ghabraie, M., Vu, K. D., Tata, L., Salmieri, S., \& Lacroix, M. (2016). Antimicrobial effect of essential oils in combinations against five bacteria and their effect on sensorial quality of ground meat. $L W T$ Food Science and Technology, 66, 332339.

Gutierrez, J., Barry-Ryan, C., \& Bourke, P. (2008). The antimicrobial efficacy of plant essential oil combinations and interactions with food ingredients. International journal of food microbiology, 124(1), 91-97.

Hammer, K. A., Carson, C. F., \& Riley, T. V. (1999). Antimicrobial activity of 
essential oils and other plant extracts.Journal of applied microbiology, 86(6), 985-990.

Holley, R. A., \& Patel, D. (2005). Improvement in shelf-life and safety of perishable foods by plant essential oils and smoke antimicrobials. Food microbiology, 22(4), 273-292.

Hsieh, P. C., Mau, J. L., \& Huang, S. H. (2001). Antimicrobial effect of various combinations of plant extracts. Food Microbiology, 18(1), 35-43.

Hyldgaard, M., Mygind, T., \& Meyer, R. L. (2012). Essential oils in food preservation: mode of action, synergies, and interactions with food matrix components. Frontiers in microbiology, 3, 12.

Ibrahium, M., El-Ghany, M. A., \& Ammar, M. (2013). Effect of clove essential oil as antioxidant and antimicrobial agent on cake shelf life. World Journal of Dairy \& Food Sciences, 8(2), 140-146.

Jayasena, D. D., \& Jo, C. (2013). Essential oils as potential antimicrobial agents in meat and meat products: A review.Trends in Food Science \& Technology, 34(2), 96-108.

Jayathunge, K. G. L. R., Kapilarathne, R. A. N. S., Thilakarathne, B. M. K. S., Fernando, M. D., Palipane, K. B., \& Prasanna, P. H. P. (2012). Development of a methodology for production of dehydrated tomato powder and study the acceptability of the product. Journal of Agricultural Technology, 8(2), 765-773.

Keeton, J. T. (1983). Effects of fat and $\mathrm{NaCl} /$ phosphate levels on the chemical and sensory properties of pork patties.Journal of Food Science, 48(3), 878-881.

Lambert, R. J. W., Skandamis, P. N., Coote, P. J., \& Nychas, G. J. (2001). A study of the minimum inhibitory concentration and mode of action of oregano essential oil, thymol and carvacrol. Journal of applied microbiology, 91(3), 453-462.

Lv, F., Liang, H., Yuan, Q., \& Li, C. (2011). In vitro antimicrobial effects and mechanism of action of selected plant essential oil combinations against four food-related microorganisms. Food Research International, 44(9), 30573064.

Nazer, A. I., Kobilinsky, A., Tholozan, J. L., \& Dubois-Brissonnet, F. (2005). Combinations of food antimicrobials at low levels to inhibit the growth of Salmonella sv. Typhimurium: a synergistic effect?. Food Microbiology, 22(5), 391-398.

Oussalah, M., Caillet, S., Saucier, L., \& Lacroix, M. (2007). Inhibitory effects of selected plant essential oils on the growth of four pathogenic bacteria: E. coli O157: H7, Salmonella typhimurium, Staphylococcus aureus and Listeria monocytogenes. Food control, 18(5), 414-420.

Pesavento, G., Calonico, C., Bilia, A. R., Barnabei, M., Calesini, F., Addona, R., ... \& Nostro, A. L. (2015). Antibacterial activity of Oregano, Rosmarinus and Thymus essential oils against Staphylococcus aureus and Listeria monocytogenes in beef meatballs. Food Control, 54, 188-199.

Radaelli, M., Silva, B. P. D., Weidlich, L., Hoehne, L., Flach, A., Costa, L. A. M. A. D., \& Ethur, E. M. (2016). Antimicrobial activities of six essential oils commonly used as condiments in Brazil against Clostridium perfringens. brazilian journal of microbiology, 47(2), 424-430.

Sánchez-González, L., Cháfer, M., Chiralt, A., \& González-Martínez, C. (2010). Physical properties of edible chitosan films containing bergamot essential oil and their inhibitory action on Penicillium italicum. Carbohydrate polymers, 82(2), 277-283. 
Skandamis, P. N., \& Nychas, G. J. (2001). Effect of oregano essential oil on microbiological and physico-chemical attributes of minced meat stored in air and modified atmospheres. Journal of Applied Microbiology,91(6), 10111022.

Thanissery, R., \& Smith, D. P. (2014). Marinade with thyme and orange oils reduces Salmonella Enteritidis and Campylobacter coli on inoculated broiler breast fillets and whole wings. Poultry science, 93(5), 1258-1262.

Thomas, R., Anjaneyulu, A. S. R., \& Kondaiah, N. (2010). Quality of hurdle treated pork sausages during refrigerated $(4 \pm 1 \mathrm{C})$ storage. Journal of food science and technology,47(3), 266-272.

Tsigarida, E., Skandamis, P., \& Nychas, G. J. (2000). Behaviour of Listeria monocytogenes and autochthonous flora on meat stored under aerobic, vacuum and modified atmosphere packaging conditions with or without the presence of oregano essential oil at 5 C. Journal of applied microbiology, 89(6), 901-909.
Turgis, M., Vu, K. D., Dupont, C., \& Lacroix, M. (2012). Combined antimicrobial effect of essential oils and bacteriocins against foodborne pathogens and food spoilage bacteria. Food Research International, 48(2), 696-702.

Van Haute, S., Raes, K., Van Der Meeren, P., \& Sampers, I. (2016). The effect of cinnamon, oregano and thyme essential oils in marinade on the microbial shelf life of fish and meat products. Food Control, 68, 30-39.

Viuda-Martos, M., Ruiz Navajas, Y., Sánchez Zapata, E., Fernández-López, J., \& Pérez-Álvarez, J. A. (2010). Antioxidant activity of essential oils of five spice plants widely used in a Mediterranean diet. Flavour and Fragrance Journal,25(1), 13-19.J.

Zhang, Y. Wang, D.D. Pan, J.X. Cao, X.F. Shao, Y.J. Chen and C.R. Ou, Effect of black pepper essential oil on the quality of fresh pork during storage. Meat science, 117, 130-136 (2016).

\section{How to cite this article:}

Anita Arya, S.K Mendiratta, R.K.Agarwal, S.K Bharti and Pramila Umarao. 2019. Antimicrobial profile and organoleptic acceptability of some essentials oils and their blends in hurdle treated chicken meat spread. Int.J.Curr.Microbiol.App.Sci. 8(09): 2162-2177. doi: https://doi.org/10.20546/ijcmas.2019.809.250 